\begin{tabular}{|c|c|}
\hline Title & Warm-cool color-based high-speed decolorization: an empirical approach for tone mapping applications \\
\hline Author(s) & A mbal athankandy, Prasoon; Ou, Y afei; Ikebe, Masay uki \\
\hline Citation & $\begin{array}{l}\text { Journal of electronic imaging, 30(4), } 043026 \\
\text { https://doi.org/10.1117/1.JEI.30.4.043026 }\end{array}$ \\
\hline Issue Date & 2021-07 \\
\hline Doc URL & http:/hdl.handle.net/2115/82941 \\
\hline Rights & $\begin{array}{l}\text { Copyright } 2021 \text { Society of Photo-Optical Instrumentation Engineers (SPIE). One print or electronic copy may be made } \\
\text { for personal use only. Systematic reproduction and distribution, duplication of any material in this publication for a fee } \\
\text { or for commercial purposes, and modification of the contents of the publication are prohibited. }\end{array}$ \\
\hline Type & article (author version) \\
\hline File Information & Our final manuscript.pdf \\
\hline
\end{tabular}

Instructions for use 


\title{
Warm-cool color-based high-speed decolorization: an empirical approach for tone mapping applications
}

\author{
Prasoon Ambalathankandy $^{\mathrm{a}, \mathrm{b}}$, Yafei Ou ${ }^{\mathrm{a}, \mathrm{b}}$, Masayuki Ikebe $^{\mathrm{a}}$ \\ ${ }^{\mathrm{a}}$ Research Center for Integrated Quantum Electronics, Hokkaido University, Sapporo 060-0813, Japan \\ ${ }^{\mathrm{b}}$ Graduate School of Information Science and Technology, Hokkaido University, Sapporo 060-0814, Japan
}

\begin{abstract}
Grayscale images are fundamental to many image processing applications like data compression, feature extraction, printing and tone mapping. However, some image information is lost when converting from color to grayscale. In this paper, we propose a light-weight and high-speed image decolorization method based on human perception of color temperatures. Chromatic aberration results from differential refraction of light depending on its wavelength. It causes some rays corresponding to cooler colors (like blue, green) to converge before the warmer colors (like red, orange). This phenomena creates a perception of warm colors "advancing" toward the eye, while the cool colors to be "receding" away. In this proposed color to gray conversion model, we implement a weighted blending function to combine red (perceived warm) and blue (perceived cool) channel. Our main contribution is threefold: First, we implement a high-speed color processing method using exact pixel by pixel processing, and we report a $5.7 \times$ speed up when compared to other new algorithms. Second, our optimal color conversion method produces luminance in images that are comparable to other state of the art methods which we quantified using the objective metrics (E-score and C2G-SSIM) and subjective user studies (decolorization and tone mapping). Third, we demonstrate that an effective luminance distribution can be achieved using our algorithm by using global and local tone mapping applications.
\end{abstract}

Keywords: Warm-cool colors, chromatic aberration, decolorization, luminance, pre-processing, RGB, tonemap. prasoon.ak@ist.hokudai.ac.jp ou.yafei.5w@ist.hokudai.ac.jp ikebe@ist.hokudai.ac.jp

\section{Introduction}

Grayscale channels, which reflect image luminance, are used for various applications such as printing, tone mapping, data compression, and feature extraction. Thus, obtaining luminance along with human perception has a key role for decolorization, which converts RGB channels to high-quality gray ones. For example, High Dynamic Range (HDR) compression is ideally performed by tone mapping the luminance channel for the lower computational and memory cost. However, applying well-known luminance channels such as $\mathrm{Y}$ of $\mathrm{YCbCr}$ or V of HSV does not guarantee appropriate tone mapping, as these channels do not reflect human perceptions. Therefore, decolorization has gathered considerable attention and various sophisticated methods to achieve perceptual decolorization have recently been proposed. These methods can be classified into global and local 
methods. Global methods can define only one conversion function for all pixels, and most of these methods use all pixels in the image to determine the function. On the other hand, local ones process the target from neighboring pixels in the same way as a spatial filter, the function is different for each pixel. However, both types of methods face the issue of calculation cost, which comes from optimization iterations or spatial filter processing.

We have developed a fast decolorization method that reflects the perception of warm and cool colors which is well known in psychophysics studies. ${ }^{1}$ Colors are arranged according to their wavelengths on the color wheel, the ones with longest wavelengths are on the right side of the wheel and are known as warm colors, as they evoke warmth. These hues include shades of red, yellow, and orange. On the other hand, green, blue and violet which have shorter wavelengths are placed on the left side of the color wheel, and are perceived as cool colors. The color of an object in a scene affects our perception of its apparent depth and this phenomenon has been exploited by many artists. This optical illusion has been studied by psychologists, and early researchers explored color-depth relationship. One of the widely accepted theory explains this phenomenon is due to fact that shorter wavelengths of visible light are refracted more than longer wavelengths. ${ }^{2}$ In other words, an equidistant source of different wavelengths cannot be focused simultaneously onto our retina. This phenomena is called as chromatic aberration and we discuss it in detail in section 2.3. In our decolorization method, we implement a weighted blending of warm and cool colors in accordance with the Helmholtz-Kohlrausch (H-K) effect. ${ }^{3}$ On that account, we make two assumptions, which are: $(i)$ warm colors (mainly including $R$ ) are lighter than $Y$ of $\mathrm{YCbCr}$ and (ii) mixed colors are darker than the $Y$ or $L$ of CIE with the same luminance. To satisfy these assumptions, we use a weighted blending of RGB channels and remap them to warm/cool colors on the luminance channel. Following are our main contributions: 


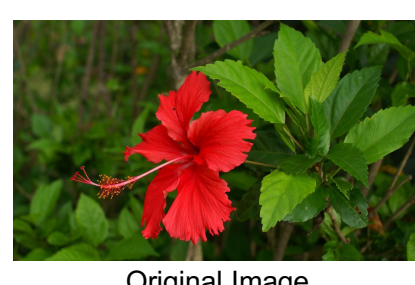

Original Image
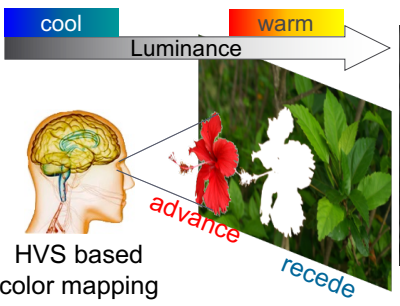

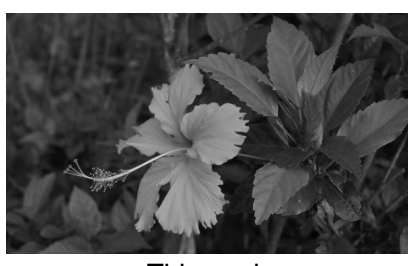

This work

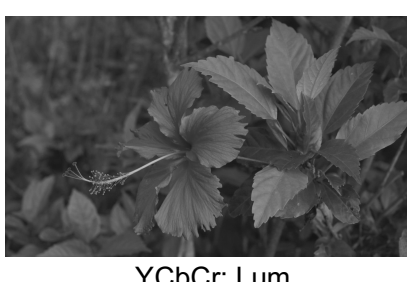

YCbCr: Lum.

Fig 1 Main concept of our decolorization method: Human perception of warm and cool colors. Warm colors "advance" toward the eye, while cool colors "recede". In this work we are able to accurately reflect the human perception of warm-cool colors, whereas this phenomenon is non-existent in conventional YCbCr color space.

- We propose a warm-cool color-based decolorization method.

- We achieve high-speed color mapping by exact pixel-by-pixel processing.

- We obtain luminance comparable to that of optimization-based methods.

- We demonstrate effective luminance distribution for pre-processing by performing objective and subjective evaluations.

There are many well defined methods to convert any color image to a grayscale image. An effortless procedure is to assign different weights to color channels, in order to have the same luminance in the grayscale image as the original color image. For example, in the MATLAB function rgb2gray, it converts any RGB values to grayscale (Gray) values by forming a weighted sum of the $R, G$, and $B$ components as Gray $=0.2989 \times R+0.5870 \times G+0.1140 \times B$. This function operates under an assumption that human visual system is more sensitive to green color. When operating with CIELab and YUV color spaces, one could directly obtain luminance channel as the grayscale version of the color image as they consider the luminance and color channel to be independent. But, such crude approaches will fail to preserve image contrast as shown in these examples (see Fig. 1 and Fig. 2). 


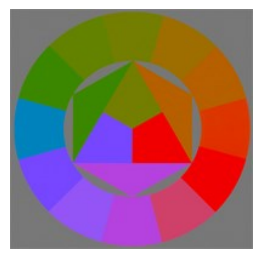

Original Image

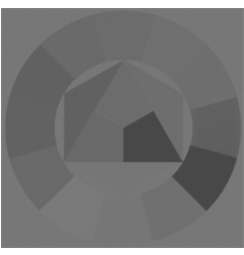

$\mathrm{Y}$ of $\mathrm{YCbCr}$

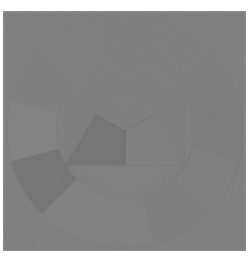

CIE Lab

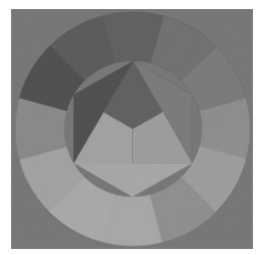

This work

Fig 2 Comparison of luminance components obtained using YCbCr, CIELAB and our proposed method. Here, we can observe that our decolorization can generate warm colors (like $R$ ) which are lighter than $Y$ of $\mathrm{YCbCr}$, and mixed colors which are darker than the $Y$ or $L$ of CIE.

In several real-world image/video processing applications like detail enhancement, image matching, and segmentation under different illumintion a 1-D grayscale image has to be obtained from its corresponding 3-D color image. However, mapping the 3-D color information onto a 1-D grayscale image while retaining the original contrast and fine details is a challenging problem. Additionally, implementing decolorization algorithms with a reasonable computational efficiency is pivotal for realising their real-time applications. Many studies have been carried out to develop novel decolorization methods. These mapping methods can be categorized into global ${ }^{4-8}$ and local methods. ${ }^{9-12}$ In local mapping methods, the same color pixel within an image could be mapped into different grayscale values depending on its spatial location. Ideally this undesirable as such output images may be perceived as unnatural. On the other hand, in global mapping methods same color pixels within an image irrespective of its spatial location are mapped to same grayscale values. Thus, global methods are more likely to produce grayscale images that are perceived to appear natural.

In the global methods category, Gooch et al. ${ }^{4}$ proposed a global decolorization algorithm that can be implemented by solving the optimization problem for all image pixels. Then, Kim et al. ${ }^{7}$ aimed at high-speed processing by simplifying Gooch's method. Smith et al. ${ }^{6}$ used unsharp masking and the $\mathrm{H}-\mathrm{K}$ effect model of Nayatani et al. ${ }^{3}$ Nayatani's model ${ }^{3}$ is merely an experimental 
model for the effect of the CIELUV chrominance component for human perception. On the other hand, the method of Lu et al. ${ }^{8}$ is focused on converting a color image into a gray image with high contrast. The main advantage of these methods is transformation consistency, i.e., the same color is converted to the same grayscale. However, speed remains a problem for these methods. Most of the local methods are aimed at speeding up the method of Lu et al. ${ }^{8}$ To enhance image contrast, Ancuti et al. ${ }^{10}$ adopted the strategy of using a Laplacian filter and Song et al. ${ }^{12}$ used a Sobel filter. Although local methods are effective in terms of contrast emphasis, they are disadvantageous in terms of tone mapping because conversion consistency is not maintained and it differs from human perception.

Recently, some machine learning-based techniques have also been proposed for image decolorization. ${ }^{13-18}$ Cai et al. ${ }^{14}$ proposed a method, that used the perceptual loss function to pretrain VGG-Net. ${ }^{19}$ However, it is difficult to control and many of their output images are far from human perception additionally, the computational cost are high. Processing an image of $256 \times 256$ size it requires roughly 30 seconds on a single Nvidia GeForceGTX 1080 GPU. Zhang et al., proposed a CNN framework that combines local and global image features. ${ }^{17}$ However, their network framework do not account for exposure features. ${ }^{13}$ Lin et al.'s method ${ }^{16}$ by utilizing a database of 50 images from the Corel dataset produced 50 grayscale images using the Color2Gray algorithm. ${ }^{4}$ With these 50 input/output image pairs as training examples for their partial differential equations-based (PDE) learning system, they learn Color2Gray mapping. The proposed PDE system generated images of comparable quality to that of Gooch et al ${ }^{4}$ However, for an input image of size $n \times n$ their PDE color mapping algorithm's computational complexity is $O\left(n^{2}\right)$. Liu and Leung proposed a deep learning method for the multiexposure fusion problem and applied for color to gray conversion using convolutional neural network $(\mathrm{CNN}) .{ }^{18}$ This paper describes the re- 
lationship between color-to-gray transformation and multiexposure fusion (MEF) and applied the CNN to the MEF. Their method has resulted in improvements in the image fusion effects, however, the cost of the processing is high and report that a $750 \times 599$ color image computation time is reported as 27.964 seconds on CPU and 1.632 seconds on GPU. ${ }^{18}$ Under these circumstances, a high-speed method for generating grayscale images that accurately captures human perception has not yet been developed. To make tone maps valid, it is necessary to develop such a method.

\section{Proposed Method}

\subsection{Problem Definition}

Luminance components such as $Y$ of $\mathrm{YCbCr}$ and $\mathrm{CIE} L$ have been used in various image processing applications; however, they do not accurately reflect human perception (Fig.1 and Fig. 2). Figure 1 shows how the warm colored flower (red) advances towards the eye of the observer, while the background mainly green recedes. Using the luminance channel of the conventional $\mathrm{YCbCr}$ color space this phenomenon is absent. But, in our method we are able to capture the warmth $R$ (red) component as perceived in human perception. Figure 2 shows that the $R$ (red) and $B$ (blue) components do not come even close to the perception in the luminance component of CIELAB. Moreover, mixed color components such as mud yellow tend to appear dark for people. In this study, we conceived the idea that RGB weight functions for alpha blending can reproduce this phenomenon.

\subsection{Luminance mapping using red and blue weighting function}

We consider the idea of color mapping by performing a weighted blending of warm-colors and cool-colors in accordance with the H-K effect. ${ }^{3}$ And we evaluate it using the COLOR250 dataset. ${ }^{20}$ 


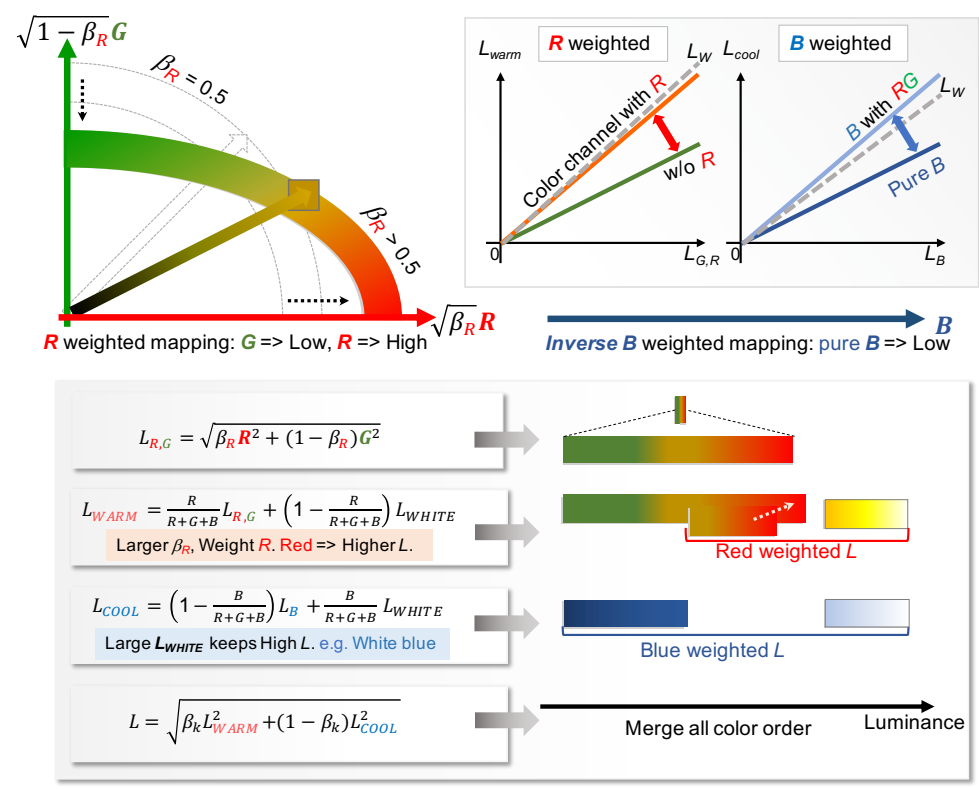

Fig 3 Remodeling of luminance space based on our two weighting functions one each for warm and cool colors. Here, luminance is defined as the Euclidean distance of warm/cool color. The included color panels aid in intuitive understanding of the proposed method.

Psychophysical studies find that, warm and cool colors impact our visual perception of the objects that we see. For example, the red color associated with fire/sun advances toward the eye, creates an illusion of heat and therefore perceived as warmth and comforting. On the other hand, cool colors have reverse effects of warm colors. Receding from the eye of the observer, cool colors reminds of the earthy objects, like meadows and oceans. These hues often are perceived as cool and refreshing ${ }^{1} \cdot{ }^{21}$ In our decolorization method, we developed two weighting functions as shown in Fig 3. One function for remapping warm colors and the other for remapping cool colors. In our method, actual luminance is defined as the Euclidean distance of weighted warm/cool luminance including the $W$ (white) channel. Essential luminance is given by

$$
L_{W H I T E}=\sqrt{\frac{R^{2}+G^{2}+B^{2}}{3}}
$$

$$
L_{B}=B
$$




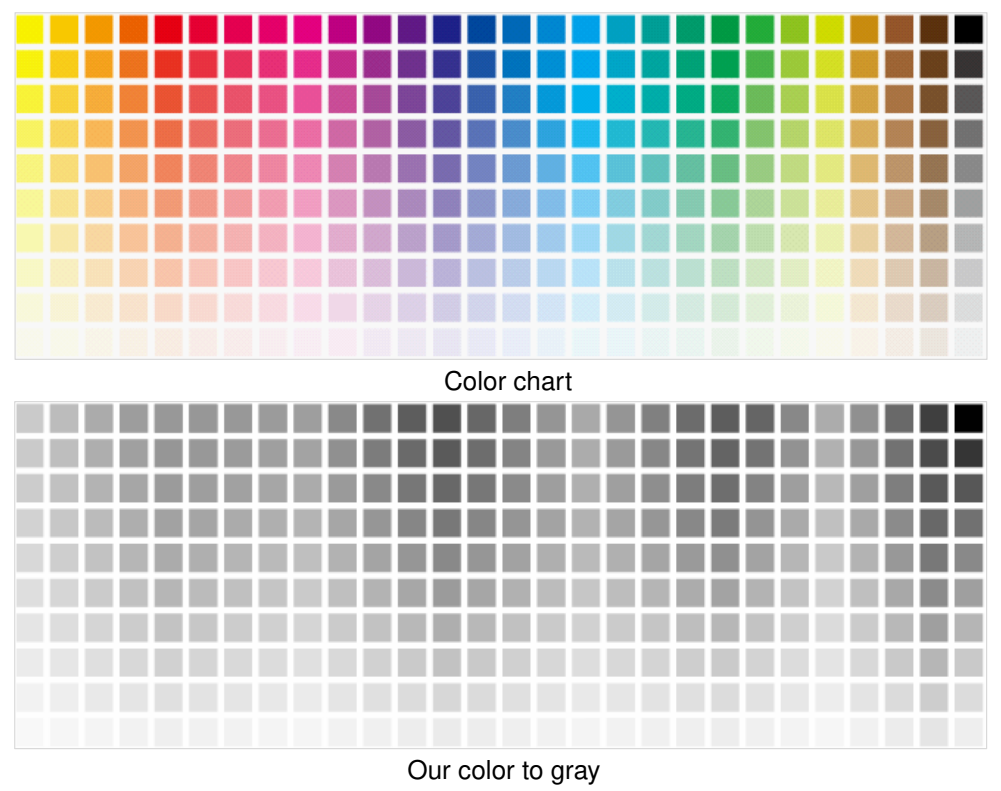

Fig 4 Decolorization of a color chart illustrating our method's effectiveness in generating brighter shades of gray for warm colors.

140

$$
L_{R, G}=\sqrt{\beta_{R} R^{2}+\left(1-\beta_{R}\right) G^{2}},\left(0.5<\beta_{R}<1\right)
$$

As we know, $L_{W H I T E}: W$ (in Eq. 1 ) is the Euclidean distance of $R G B$ channels; $L_{B}$ (in Eq. 2 ) is the $B$ (blue) channel as it is. The component of warm color function $L_{R, G}$ which includes red, orange and yellow is also defined as the Euclidean distance by $\beta_{R}$ weighted $R$ (red) and $G$ (green) vectors (in Eq. 3). When $\beta_{R}=0.5$, the vector length of each color is same. Here, we focus on the relationship between red and green as in $\mathrm{H}-\mathrm{K}$ effect; green has less brightness than red with same luminance. Thus, we apply a weight $\beta_{R}>0.5$ as a bias for the $R$ component; when $\beta_{R}$ increases, more $R$ components than $G$ components are rated. In actual $\mathrm{H}-\mathrm{K}$ effect, color brightness order is yellow, green and red $(\mathrm{Y}, \mathrm{G}, \mathrm{R}){ }^{3}$ However, since yellow is generated by mixing of red and green, if we manually place yellow as darker shade than green, the vector length of yellow color becomes similar to the length of green vector. Thus, we keep the color brightness order as green, yellow and red $(\mathrm{G}, \mathrm{Y}, \mathrm{R})$. The remappings made by the blending function using the $R$ and $B$ ratio of $R G B$ 


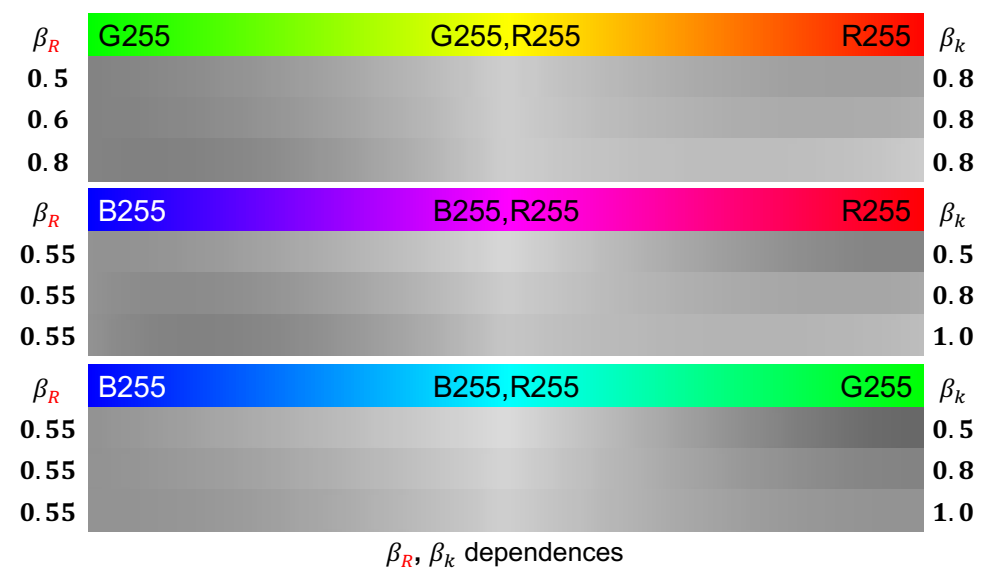

Fig 5 Impact of $\beta_{R}$ and $\beta_{k}$ on output luminance value. (top) $\beta_{k}$ set to 0.8 and for changing $\beta_{R}$ we move from $G_{\max }$ to $R_{\max }$. (middle) $\beta_{R}$ set to 0.55 and for changing $\beta_{k}$ we move from $B_{\max }$ to $R_{\max }$. (bottom) $\beta_{R}$ set to 0.55 and for changing $\beta_{k}$ we move from $B_{\max }$ to $G_{\max }$

154

values are given by

$$
L_{W A R M}=\frac{R}{R+G+B} \cdot L_{G, R}+\left(1-\frac{R}{R+G+B}\right) \cdot L_{W H I T E}
$$

$$
L_{C O O L}=\left(1-\frac{B}{R+G+B}\right) \cdot L_{B}+\frac{B}{R+G+B} \cdot L_{W H I T E}
$$

Here, $L_{W A R M}$ is obtained by blending red weighted $L_{R, G}$ with white as shown in Fig. 3 and Eq. 4. The blending ratio determines the relationship of warm color components and the colors which are closer to white. When the color includes large red components (e.g. pure red), this color has large brightness approaching white. In inverse $B$ weighting, pure $B$ components are assigned to low luminance in the $L_{C O O L}$. Since both functions are blended with $L_{W H I T E}$, bright orange/yellow and sky blue, which include high white components, are mapped to higher luminance. Finally, we obtain the luminance channel $L$, which is given by

$$
L=\sqrt{\beta_{k} L_{W A R M}^{2}+\left(1-\beta_{k}\right) L_{C O O L}^{2}}\left(0.5<\beta_{k}<1\right)
$$

In this study, we mainly used warm-color weighting luminance in experiments and set the $\beta_{k}$ 
higher. We set two parameters relating to color component emphasis as follows: $\left(\beta_{R}=0.55 ; \beta_{k}=\right.$ 0.8 ), and from Fig 5 it can be easily understood how $\beta_{R}$ and $\beta_{k}$ can bias the resulting luminance values. Figure 4 presents a color reference chart and the corresponding grayscale conversion intended for visual comparisons and measurements. This chart illustrates our method's effectiveness in generating brighter shades of gray for warm colors.

\subsection{Warm-cool color and chromatic aberration}

From Snell's law we know that the refraction of light is dependent on its wavelength. As the frequency of light increases, its refractive index becomes larger, causing more refraction of the shorter wavelengths. Therefore, when an image is captured through a lens, all colors do not focus at the same distance, and these imperfections are known as chromatic aberration. In cameras this imperfection is removed by using a combination of second achromatic lens which is made of different material(glass) than the first lens. This second lens would reverse the color dispersion caused by the first lens. The human eye, also employs a lens and does exhibit this phenomena as shown in Fig. 6. From this figure we can observe that red light forms the image farthest from the lens as it has the smallest refractive index. Colors with higher index of refraction would ideally bend more thereby forming images closer to the lens. Therefore, it would be impossible to focus on all colors simultaneously, resulting in "somewhat fuzzy" images that are not in focus. Colors that are closer to red end of the electromagnetic spectrum are said to be warm colors and are perceived as closer to the observer. ${ }^{22,23}$ Colors that are around the blue end are said to be cooler colors and are perceived to be receding away from the observer. This phenomenon has been exploited by traditional artists to add depth information in artwork, ${ }^{24}$ display devices,,${ }^{25}$ and $3 \mathrm{D}$ imagery. ${ }^{26}$ 


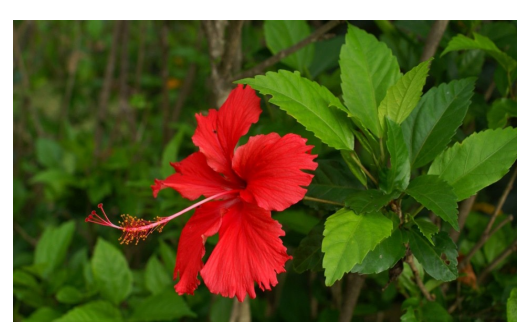

Original Image
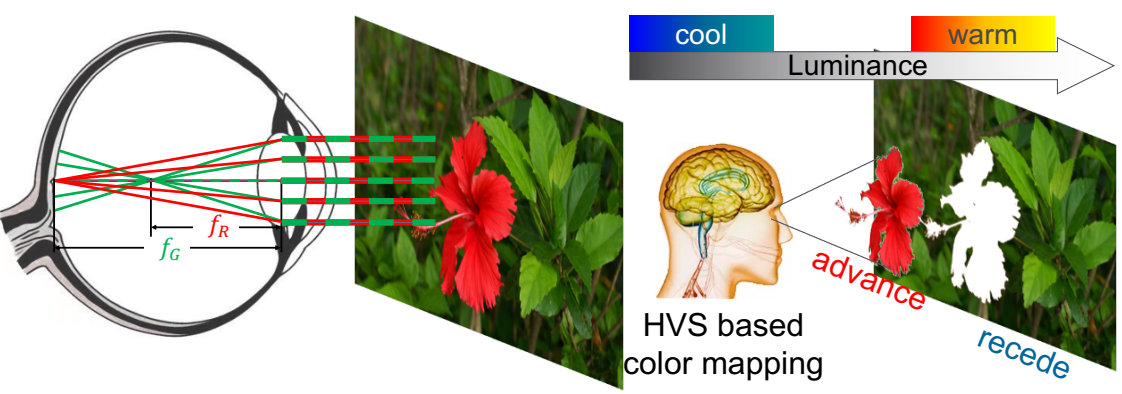

Fig 6 Chromatic aberration which results from differential refraction of light depending on its wavelength, it causes some rays (green) to converge before other (red). This results in a perception of red "advancing" toward the eye, while green to be "receding".

\subsection{Limitation of our method}

In our decolorization method, pure green is likely to be mapped to a dark luminance value. Light green will also be mapped to a lesser dark luminance part as shown in Fig. 5. Therefore, certain scenes are likely to be perceived as unnatural. For example: (a) Vegetables (e.g. leafy greens like cabbage) (b) Green meadows under bright sky. (c) Bright green neon lights. However, in Fig. 7 we perceive them as natural. We postulate the following as the possible reasons: (i) There are rarely any pure bright green (like G255) scene in nature. (ii) In our color space, green color with white components follow Eq.(1) by weighting function. Therefore, the color keeps a balance among other color channels. (iii) Vegetation scenery with dark green are well perceived as healthy plants.

\section{Experimental Results}

\subsection{Comparison with related decolorization methods}

When evaluating our method, we focused on the following points:

- Objective and subjective image quality assessment.

- Compare their processing speeds. 

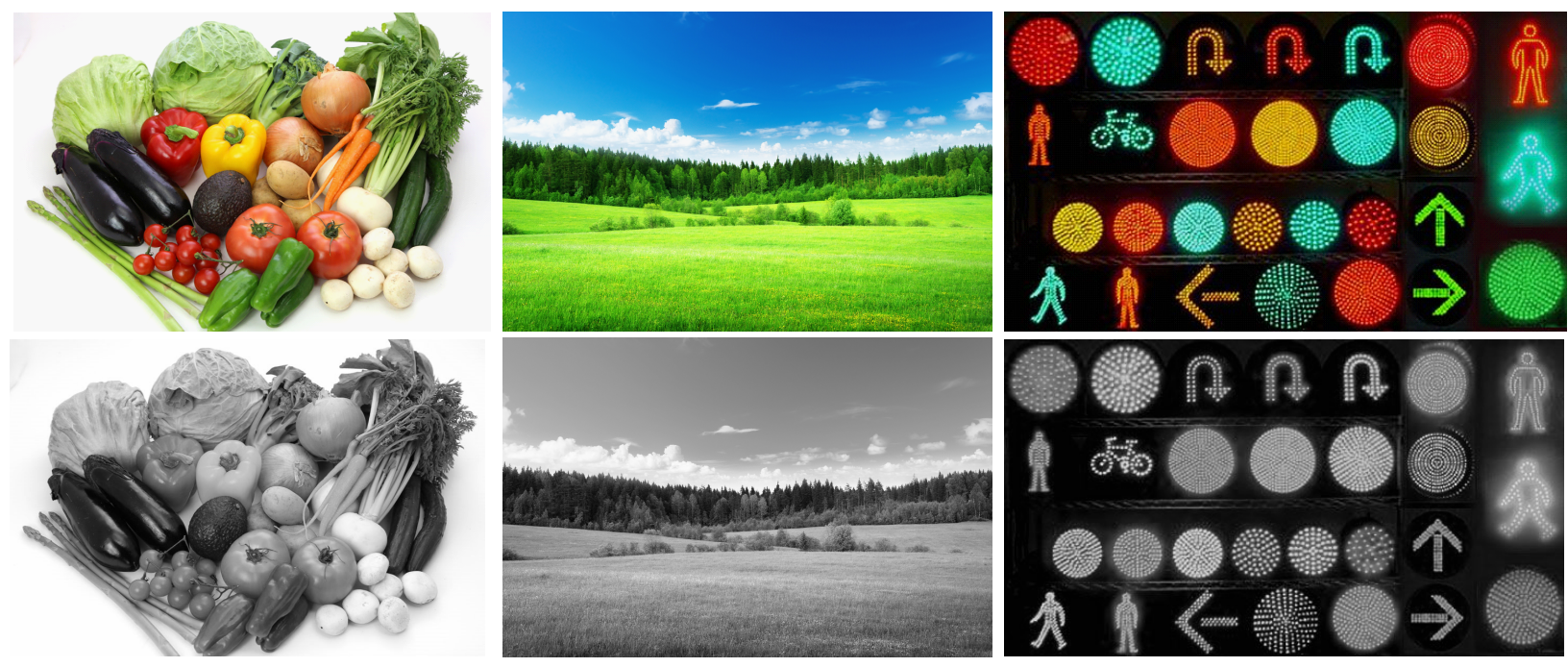

Fig 7 Examples to demonstrate limitation of our decolorization method when mapping bright green/neon color.

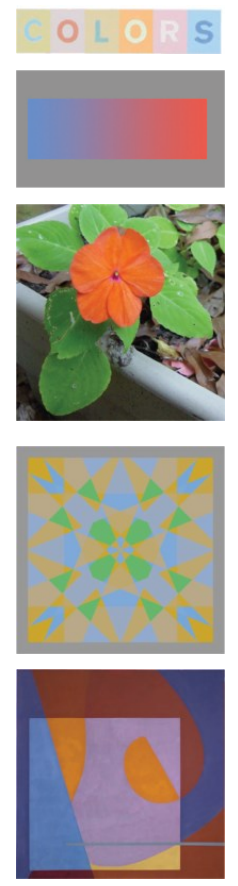

Original Images
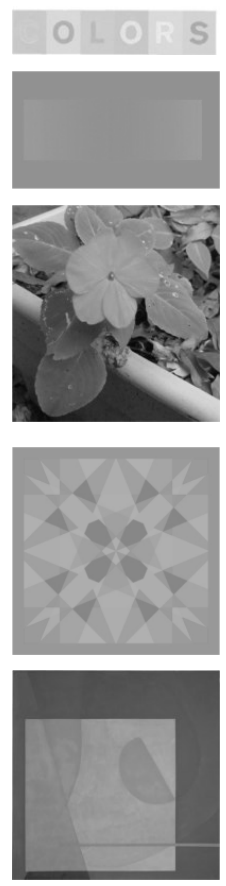

Ours
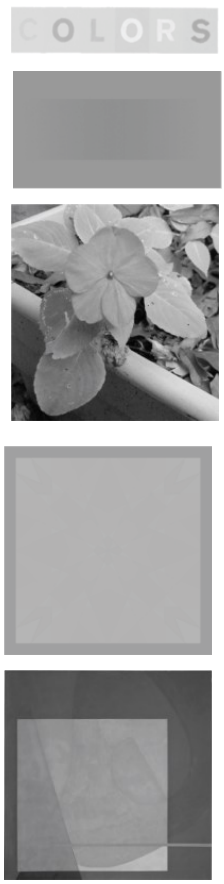

L of CIE Lab
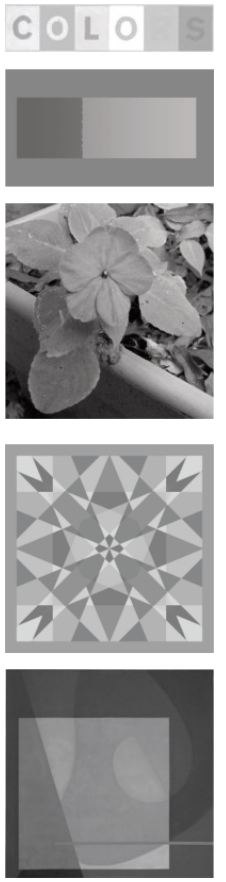

Gooch et al.
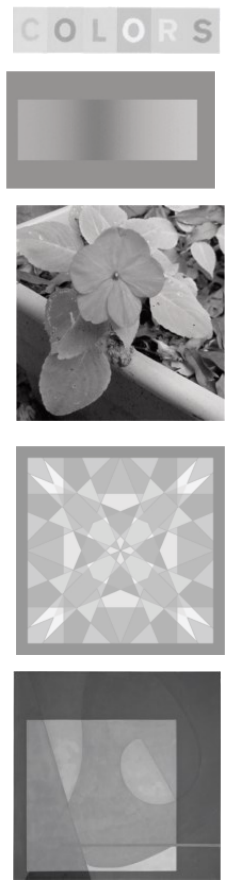

Kim et al.
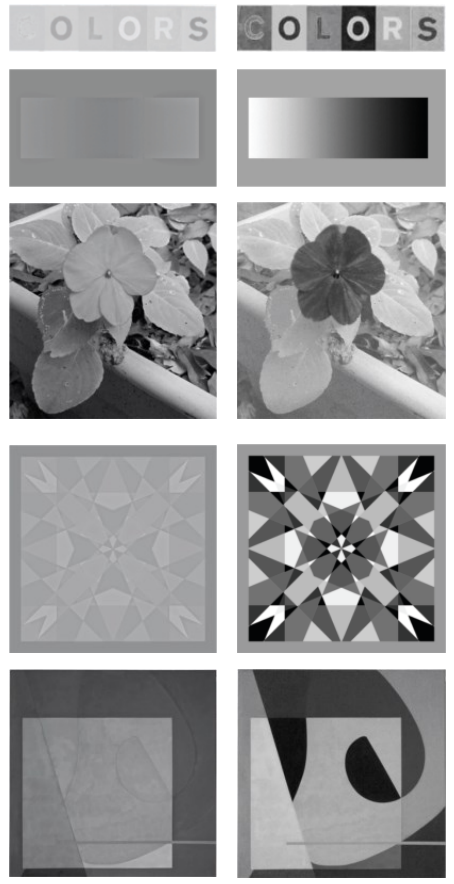

Smith et al.

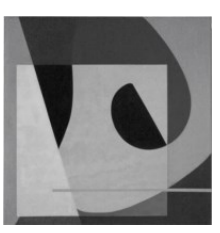

Lu et al.

Fig 8 Color to gray conversion comparison with other decolorization methods.

The evaluations demonstrated that our method does reflect human perception better than or equal to other optimized methods (refer sections 3.2 and 4.3). It delivers high-speed processing, and is a useful tool for many image processing applications. Figure 8 shows a comparison between our proposed method and other decolorization methods, and a detailed subjective evaluation is 
presented in section 3.3. For this comparison we have used images from Cadik's dataset. ${ }^{27}$ In Fig. 8 first and second columns from the left are original images and images obtained with our method respectively. The images in the third column from the left were obtained with the $L$ component of the CIELAB color space, which is a reversible model reflecting human visual characteristics. The images in columns four, five and six were obtained from global decolorization methods, which means only one conversion function is applied to each pixel. For example, in these methods, optimization techniques referring to whole pixels are applied to luminance conversion without regard to the brightness perceived by human perception. The images in the far right column were obtained with a local method that refers to pixel values in the local patch of the image for contrast enhancement.

In the $L$ component of the CIELAB color space, the base luminance is $Y$ in the $\mathrm{YCbCr}$ color space; the luminance is also mapped along with the color order of $Y$. In the image (third column, fourth row), since the luminance of all colors are the same in $Y$, output values are also the same in the CIELAB color space. However, we can perceive the contrast in this image. Thus, an appropriate conversion is required; our method can generate the perceived contrast in this image. Gooch et al. ${ }^{4}$ obtained the highest average C2G-SSIM score but clearly a step artifact occurs in the gradation image (fourth column, second row). Kim et al. ${ }^{7}$ proposed an improved version of Gooch's method that achieves high-speed optimization and reflects the H-K effect. ${ }^{3}$ In this method, since a weighting function is applied for expanding luminance distribution of whole pixel colors in the image along with chrominance, its conversion becomes different in each image. Thus, overenhancement is observed in the images (fifth column, first row) and (fifth column, fourth row). The output images of our method are similar to those of Smith et al. ${ }^{6}$ Their method uses the H-K effect, but it also requires a lot of processing time for post-unsharp-mask filtering. In Lu et al., ${ }^{8}$ 
Table 1 Run-time comparison table with other decolorization algorithms.

\begin{tabular}{|c|c|c|c|c|c|c|}
\hline Algorithm & Processing time & Image size $\mathrm{W} \times \mathrm{H}$ & CPU Clock Speed & Optimization & Process & Normalized Time ${ }^{\star}$ \\
\hline Gooch et al. ${ }^{4}$ & $25.7 \mathrm{~s}$ & $200 \times 200$ & -GPU- & $\checkmark$ & Global & N/A \\
\hline Kim et al. ${ }^{7}$ & $102 \mathrm{~ms}$ & $320 \times 240$ & $2.66 \mathrm{GHz}$ & $\checkmark$ & Global & $1.30 \mu \mathrm{s}$ \\
\hline Smith et al. ${ }^{6}$ & $6.7 \mathrm{~s}$ & $570 \times 593$ & $3.0 \mathrm{GHz}$ & $x$ & Global+Local & $22.02 \mu \mathrm{s}$ \\
\hline Lu et al. ${ }^{8}$ & $800 \mathrm{~ms}$ & $600 \times 600$ & $3.80 \mathrm{GHz}$ & $\checkmark$ & Global Contrast & $3.12 \mu \mathrm{s}$ \\
\hline Song et al. ${ }^{12}$ & $40 \mathrm{~ms}$ & $320 \times 240$ & N/A & $x$ & Local Contrast & N/A \\
\hline Ancuti et al. ${ }^{10}$ & $100 \mathrm{~ms}$ & $800 \times 600$ & $2.5 \mathrm{GHz}$ & $x$ & Local Contrast & $0.19 \mu \mathrm{s}$ \\
\hline L of CIE Lab & $25.57 \mathrm{~ms}$ & $800 \times 600$ & $2.7 \mathrm{GHz}$ & $x$ & Global & $0.053 \mu \mathrm{s}$ \\
\hline $\begin{array}{l}\text { Ours low res } \\
\text { Ours high res }\end{array}$ & $\begin{array}{l}16.71 \mathrm{~ms} \\
202.05 \mathrm{~ms}\end{array}$ & $\begin{array}{l}800 \times 600 \\
3008 \times 2008\end{array}$ & $\begin{array}{l}2.7 \mathrm{GHz} \\
2.7 \mathrm{GHz}\end{array}$ & $\begin{array}{l}x \\
\times\end{array}$ & $\begin{array}{l}\text { Global } \\
\text { Global }\end{array}$ & $\begin{array}{l}\mathbf{0 . 0 3 4} \mu \mathrm{s} \\
\mathbf{0 . 0 3 3} \mu \mathrm{s}\end{array}$ \\
\hline
\end{tabular}

* Normalized time is the processing time normalized by frequency $(2.7 \mathrm{GHz})$ and divided by the number of pixels. It indicates the effective processing time per pixel.

their method does not reflect human perception; they try generating high contrast images for mask images that are input to an edge-preserving filter such as a guided filter.

Table 1 lists the processing speed of various methods, our proposed implementation and the CIELAB were implemented in $\mathrm{C}++$; these codes were executed on an Intel Core i5-5257U (2.70GHz) CPU without any multicore, multithread or SIMD operations. The results confirmed that our method had the fastest run-time among the methods compared. It is worth noticing that it exceeded CIELAB in runtime; this indicates it also has advantages in total calculation cost including post-processing. Its computational complexity is only $O(1)$ because it performs exact pixel by pixel processing, referring only to the RGB value at each pixel. Among other methods, the one developed by Gooch et al. was reported to have $O\left(n^{4}\right)$ computational complexity. Using $O(1)$ spatial filtering it is possible to develop $O(1)$ local methods, ${ }^{10,12}$ but the filter calculations required would degrade their run-time in comparison to our decolorization method. The proposed color to gray technique has demonstrated faster run-time than local methods by maintaining global coherence, which means the conversions were the same in all pixels. 
Table 2 Comparison of seven color to gray methods based on average objective metrics for 250 images.

\begin{tabular}{|l|l|l|l|l|l|}
\hline & Decolorization & CCFR & CCPR & E-score & C2G-SSIM \\
\hline (a) & Lu et al. $^{20}$ & 0.9922 & 0.9645 & 0.9781 & 0.8900 \\
\hline (b) & Nafchi et al. $^{30}$ & 0.9880 & 0.9555 & 0.9710 & 0.8900 \\
\hline (c) & Grundland et al $^{5}$ & 0.9811 & 0.9584 & 0.9747 & 0.8749 \\
\hline (d) & Smith et al. $^{6}$ & 0.9880 & 0.9555 & 0.9710 & 0.8935 \\
\hline (e) & Gooch et al. $^{4}$ & 0.9839 & 0.9545 & 0.9714 & 0.9062 \\
\hline (f) & Kim et al. $^{7}$ & 0.9682 & 0.9310 & 0.9047 & 0.8569 \\
\hline (g) & Ours & 0.9890 & 0.9576 & 0.9728 & 0.9018 \\
\hline
\end{tabular}

\subsection{Objective Image Quality Assessment}

In our experiments, we utilized the color 250 dataset which comprises of 250 natural and synthetic color images. ${ }^{20}$ To quantitatively evaluate our decolorization algorithm we choose two objective metrics: E-score and C2G-SSIM by Ma et al. ${ }^{28}$ E-score is a joint measure proposed by Lu et al., a harmonic mean which is computed by combining two metrics: Color Contrast Preserving Ratio (CCPR), and Color Content Fidelity Ratio (CCFR). ${ }^{20}$ The CCPR is useful in maintaining the color contrast in decolorization images which is perceivable to humans. Specifically, when the color difference is smaller than a certain threshold value, it becomes undetectable to humans. Furthermore, CCFR estimates if the decolorization image is accurate in terms of structures when compared to the original color image. C2G-SSIM is new color to gray objective evaluation metric based on the Structural Similarity (SSIM) index quality metric. ${ }^{29}$ The C2G-SSIM generates quality map and has good correlation with HVS subjective preference. Table 2 presents the average Escore and C2G-SSIM for the color 250 dataset in comparison with other decolorization methods.

In our experiment we computed the average CCPR for the 250 images in the dataset by varying $\tau$ from 1 to $15 .{ }^{20}$ As can be seen from Fig .9, our algorithm's performance is reasonable and practicable when compared to other color to gray algorithms. Figure 10 shows the average C2GSSIM score for the seven decolorization methods. According to the plot in Fig .9, Lu et al.'s method shows best performance based on the E-score, however, our method delivers high average 


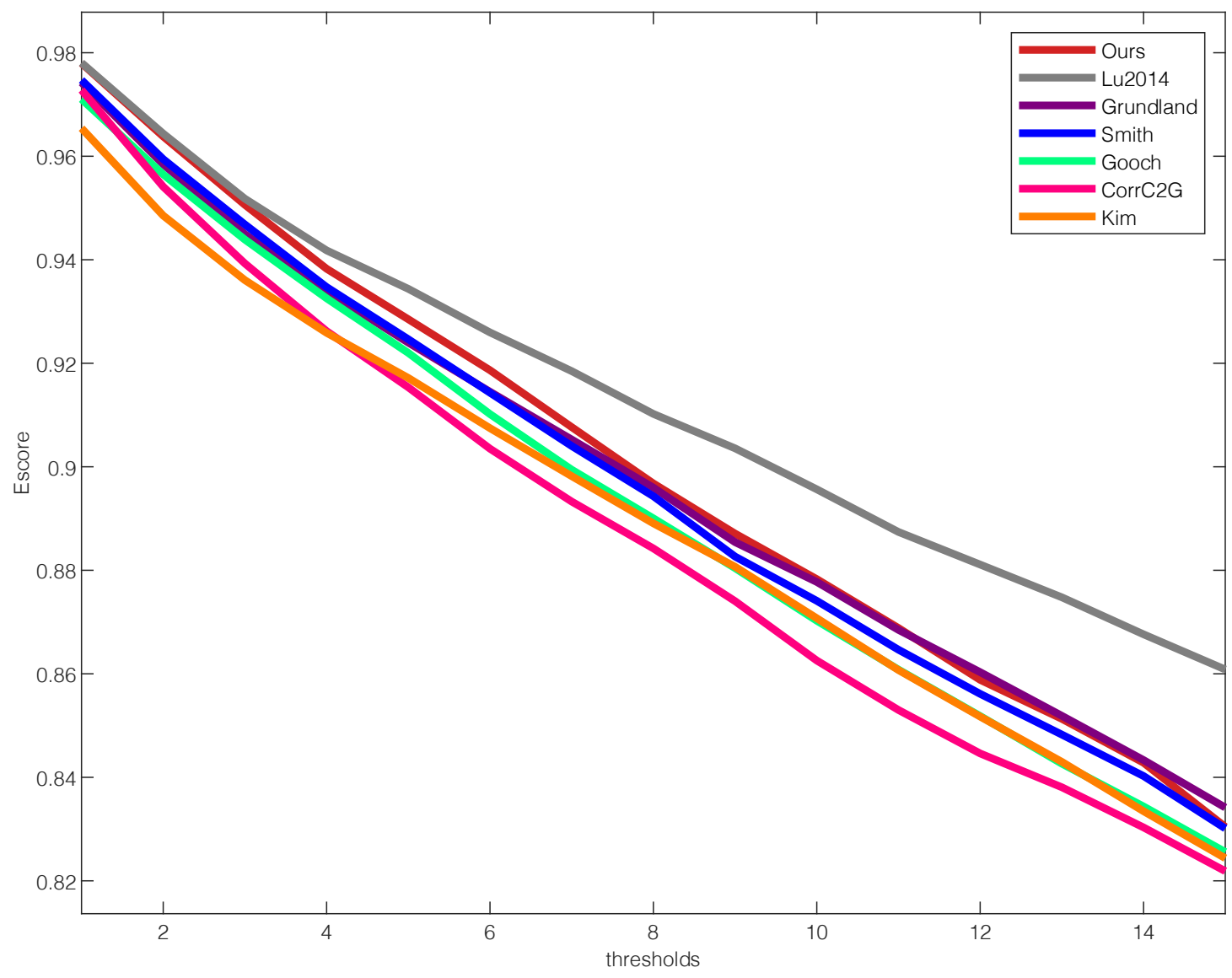

Fig 9 Comparison of seven color to gray methods based on the E-score.

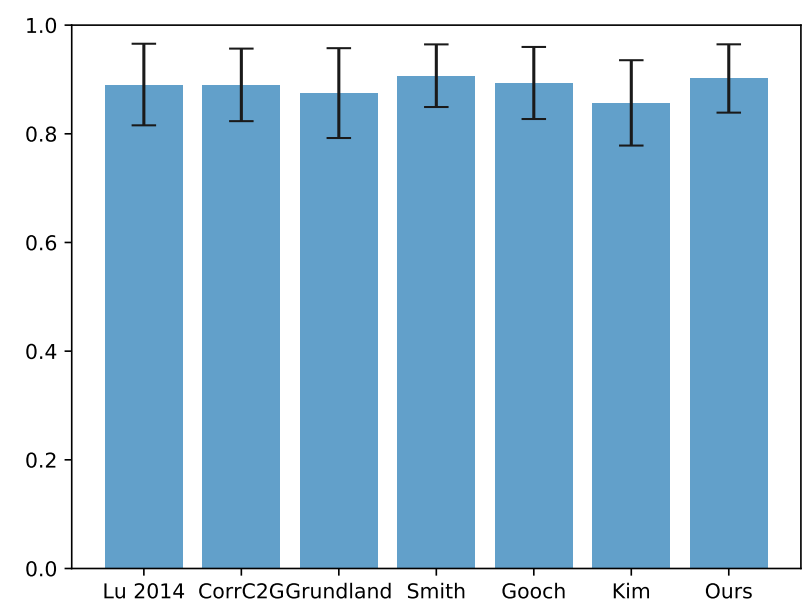

Fig 10 Comparison of seven color to gray methods based on the C2G-SSIM score.

257 C2G-SSIM measure which is better correlated to human perception. ${ }^{30}$ 


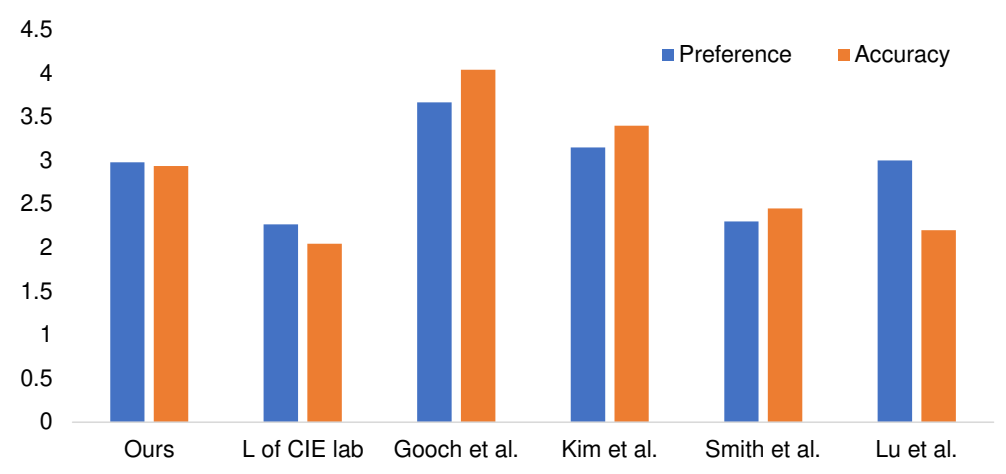

Fig 11 Subjective visual test survey and mean opinion score.

\subsection{Subjective Image Quality Assessment}

The main objective of our perceptual evaluation is to determine the accuracy and preference ${ }^{20,27}$ of our decolorization method. Our study group of 15 students $(9$ males, 6 females, average age $=$ 23) were shown five sets of images (refer Fig. 8) from Cadik's dataset. ${ }^{27}$ The group was asked to evaluate the images and assign points to them on a scale of 1 (low) to 5 (high) for accuracy and preference. For the first task, that is to measure the accuracy they compared the original color images to their decolorized output image. For the preference measurements, the user group compared the decolorized images only, i.e., without referring to the corresponding color image.

\section{Tone mapping application}

The color to grayscale conversion is a dimensionality reduction problem whose significance has been underestimated. Usually the tone mapping is performed on the grayscale image because of the lower computational and memory requirements, when compared to tone mapping on the RGB channels. In this section we will discuss global and local tone mapping application using the proposed decolorization algorithm and demonstrate its effectiveness. 

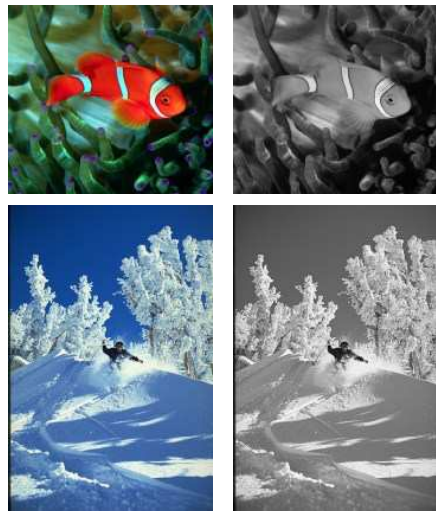

Original
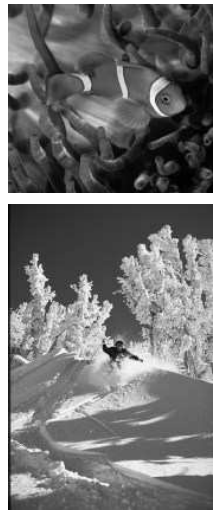

Y Lum
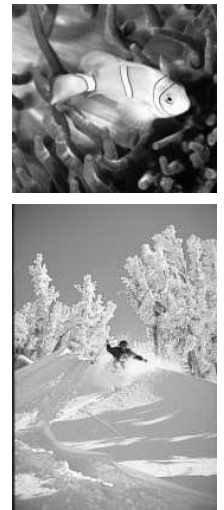

V Lum
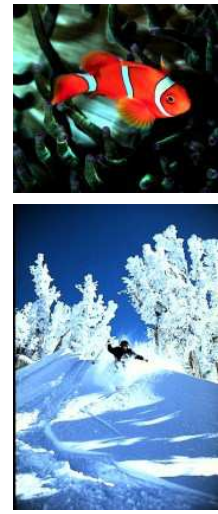

Ours Mapped
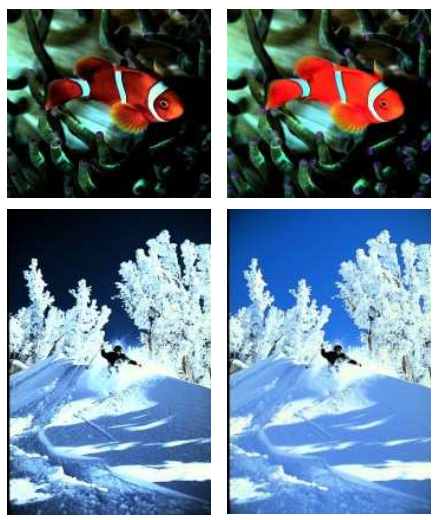

$\checkmark$ Mapped

Fig 12 Global tone mapping performed in three different luminance spaces: Ours, Y of YCbCr and V of HSV

\subsection{Global tone mapping}

We discuss our decolorization method's applicability for post-processing tasks such as tone mapping. Conventional methods have not shown such applicability because of their long run-time; thus, the use of $Y$ of $\mathrm{YCbCr}$ or $V$ of $\mathrm{HSV}$ for tone mapping is popular today. We evaluated our method's output of global tone mapping because it has the ability to adequately remap colors to $1-D$ luminance. Thus, even using global tone mapping confirms the effectiveness of our method. Figure 12 shows tone mapped results using $Y, V$, or our luminance channel. In the fish image, using $Y$, the fish color became the same as the background and could not be controlled separately, because the weight of $R$ is small in the $\mathrm{YCbCr}$ color channel. Our method was able to separate fish color from background in the same way as human perception and directly mapped the color of the background to dark. In the sky and snow scene, the global tone curve for contrast enhancement is a centered sigmoid curve, in which highlight/shadow clippings occur naturally. Since HSV color space treats primary colors and whites the same way, colors of sky and snow were mapped to luminance that was too light. Thus, the sigmoid function degraded the contrast of the outputted image. YCbCr treats $B$ as too low luminance; the sky became too dark. We confirmed that our 


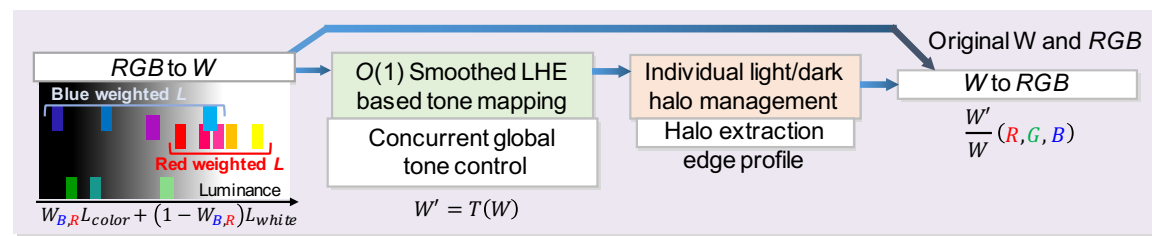

(a) Application for local TM pipeline
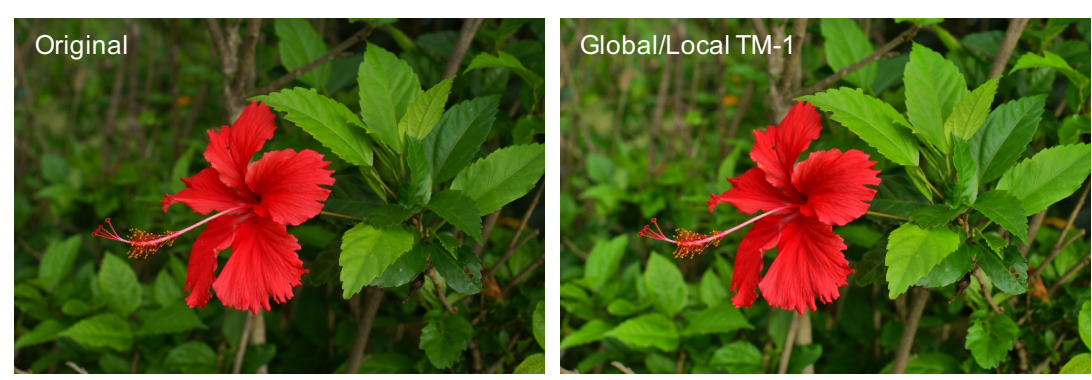

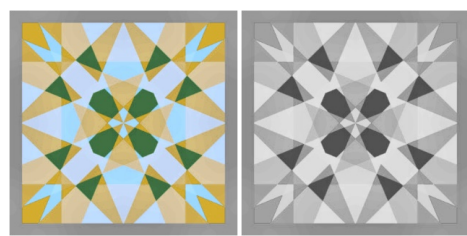

(b) Color boost with local TM

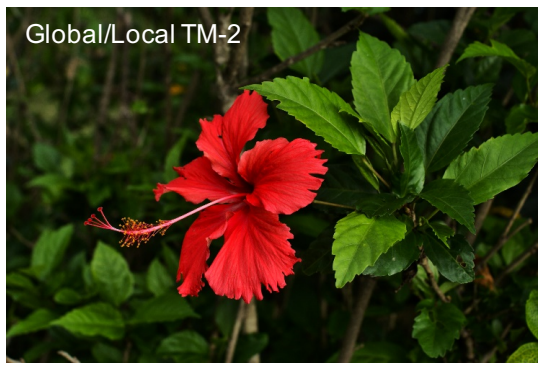

(c) Leaf/flower individual control with local TM

Fig 13 Application: (a) Ideal image processing pipeline (tone mapping) (b) Color boosted by local tone mapping and corresponding grayscale image. (c) Demonstration example for easy control features using our decolorization algorithm. TM1: background boost TM2: background suppression

method generates well-balanced images maintaining the contrast and the colors.

\subsection{Local tone mapping}

Local histogram equalization based local tone mapping converts target pixels by using tone curves constructed from local cumulative histograms. Smoothed local histogram equalization (LHE) are also used as a smoothed LH filter. ${ }^{31}$ The Apical's $\left(\mathrm{ARM}^{\circledR}\right)$ Iridix algorithm, ${ }^{32}$ which is based on smoothed LHE are used by a range of camera makers, including Nikon, Olympus and Sony. For our local tonemap application we selected smoothed LHE-based function as they are very suitable for practical real-time applications, in Fig. 13(a) we show an ideal pipeline for such a system. We implemented the local tonemap application similar to one presented by Ambalathankandy et al., ${ }^{33}$ as their implementation has a linear $O(1)$ computational complexity and produces output images with fine quality as shown in Fig. 13(b, c). The total computational time including our proposed decolorization and the local tonemap operation was only $14.7 \mu s$ per pixel (normalized time @2.7GHz CPU). This time utilization is $20 \times$ less than Lu et al.'s work. ${ }^{8}$ Additionally, using 
our decolorization method has an advantage, which is to individually control the back/foreground as shown in Fig. 13(c).

\subsection{HDR tone mapping subjective user study}

In this user study we collected response from an online survey of 50 volunteers (31 males, 19 females, average age $=29$ ). They evaluated five sets of HDR tone mapped images which are shown in Fig. 14. The main objective of this second user study is to judge the overall quality of the tone mapped images using different decolorized images which are obtained using simple histogram equalization method. Volunteers were asked to evaluate the tone mapped images for their overall perceptual quality, they rated 5 for images that were perceived as best with minimal artifacts on a scale 1 to 5. The compiled response of the user study group are presented in Fig. 15 .

\section{Conclusion}

In this paper, we present warm-cool color-based RGB to gray conversion model by taking in to account the chromatic aberration phenomena. This anomaly results from differential refraction of light depending on its wavelength, it causes some of the rays (cool colors) to converge before others (warm colors). This results in a perception of warmer colors "advancing" towards the eye, while the cooler ones to be "receding". Essentially, since decolorization is expected to have a key role in the pre-processing of tone mapping or edge preserving filters, low calculation cost and fast operation for the processing are required. To address this requirement, we have developed a high-speed $O(1)$ decolorization method that is based on warm-cool color-based perception. It refers to RGB values in one pixel and performs weighted blending of the Euclidean distances of warm/cool color vectors. This simple conversion outputs a gray channel that is comparable to the 


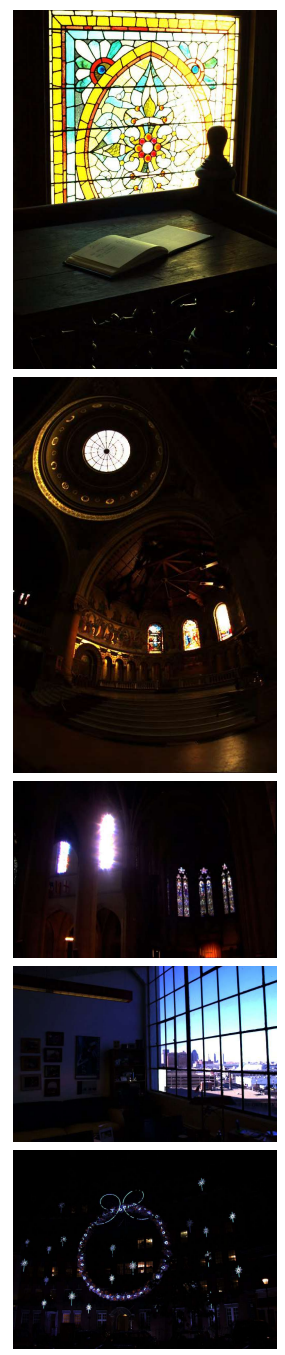

(a)
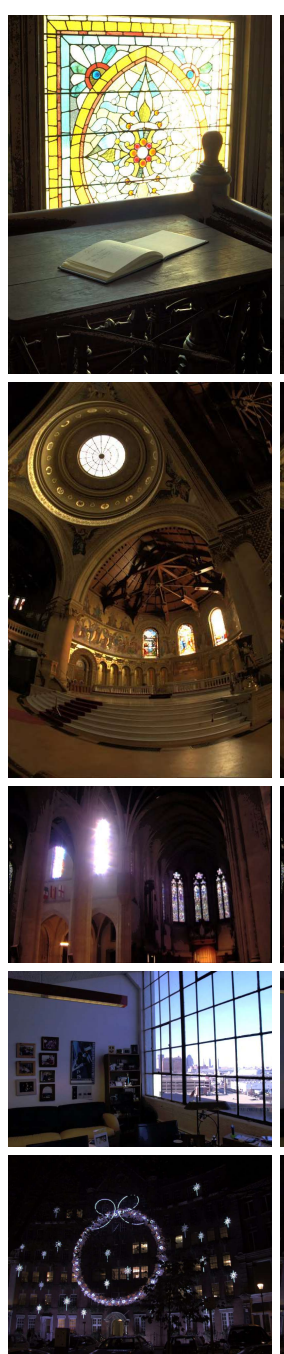

(b)
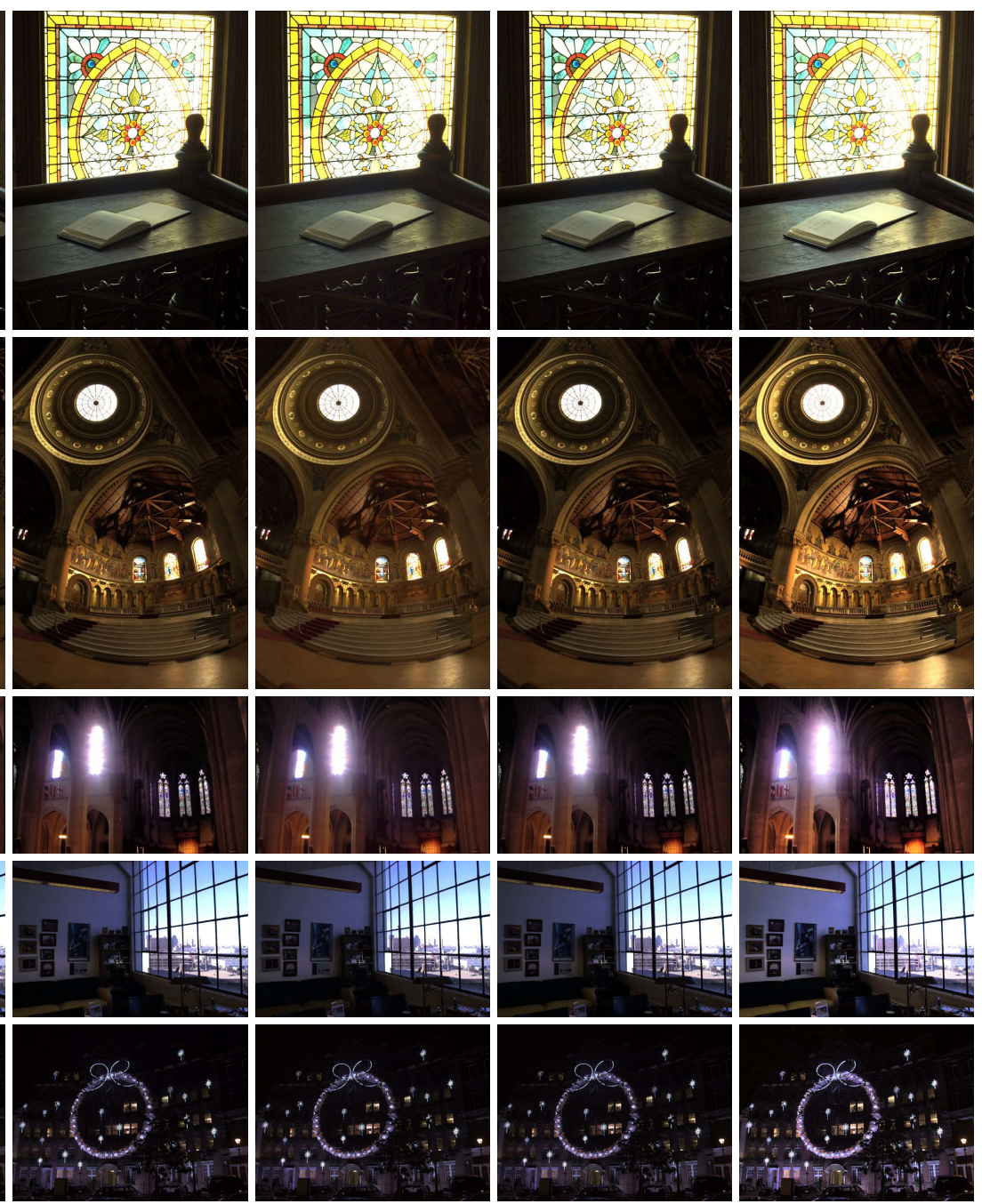

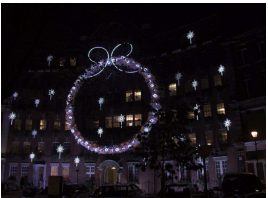

(c)

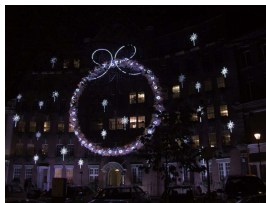

(d)

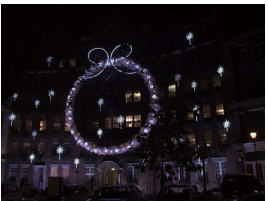

(e) (f)

Fig 14 Effect of different decolorization on HDR tone mapping using simple histogram equalization. (a) HDR test image. (b) CIELab (c) YCbCr (d) Xiong et al. ${ }^{34}$ (e) Liu et al. ${ }^{35}$ (f) Ours

conventional optimization methods using iterations. When our method is applied to tone mapping,

it achieves better results than one could be obtained with $\mathrm{YCbCr} / \mathrm{HSV}$ color space.

\subsection{Acknowledgments}

We would like to sincerely thank Cadik et al., Lu et al. for making available their datasets, and other authors for sharing their source codes. 


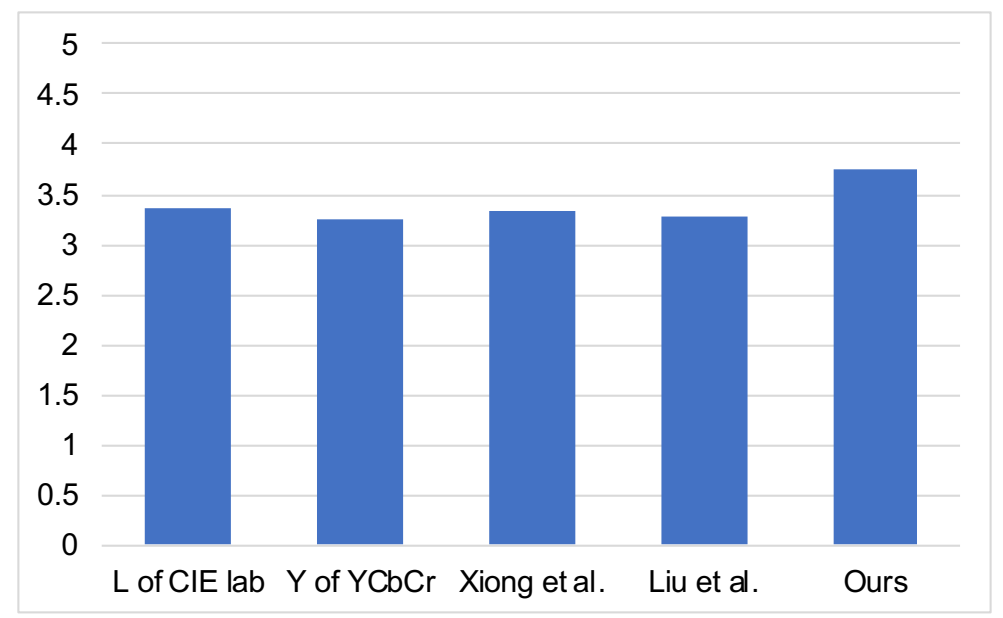

Fig 15 Mean opinion score from the tone mapping subjective user study.

\subsection{Biographies}

Prasoon Ambalathankandy is a NEDO Postdoctoral Fellow at the Research Center For Integrated Quantum Electronics (RCIQE), Hokkaido University. He received his M.Sc. degree in Electrical Engineering from the University of Calgary, Canada and Ph.D. degree in Informatics from Hokkaido University. His main topics of interest are software and hardware design for image processing systems.

Yafei Ou received his undergraduate degree in software engineering from the Chongqing University of Posts and Telecommunications, Chongqing, China in 2017. and M.S. degree in information science and technology from the Hokkaido University, Sapporo, Japan in 2020. He is currently working towards his Ph.D degree in information science and technology at the Research Center for Integrated Quantum Electronics (RCIQE), Hokkaido University, Sapporo, Japan. His research interests include computer vision, medical image processing and machine learning.

Masayuki Ikebe is a Professor and leads the Functional Communication Devices and Circuits laboratory at the Research Center For Integrated Quantum Electronics (RCIQE), Hokkaido University. His current research interests are analog and mixed-signal IC design with an emphasis on 
CMOS image sensor, medical haptic devices, THz imaging devices and intelligent image processing systems.

\section{References}

1 J. L. Morton, Color logic, Colorcom (1998).

2 J. M. SUNDET, "Effects of colour on perceived depth: review of experiments and evalutaion of theories," Scandinavian Journal of Psychology 19(1), 133-143 (1978).

3 Y. Nayatani, "Simple estimation methods for the helmholtz kohlrausch effect," Color Research \& Application 22(6), 385-401 (1997).

4 A. A. Gooch, S. C. Olsen, J. Tumblin, et al., "Color2gray: salience-preserving color removal," ACM Transactions on Graphics (TOG) 24(3), 634-639 (2005).

5 M. Grundland and N. A. Dodgson, "Decolorize: Fast, contrast enhancing, color to grayscale conversion," Pattern Recognition 40(11), 2891-2896 (2007).

6 K. Smith, P.-E. Landes, J. Thollot, et al., "Apparent greyscale: A simple and fast conversion to perceptually accurate images and video," in Computer Graphics Forum, 27(2), 193-200, Wiley Online Library (2008).

7 Y. Kim, C. Jang, J. Demouth, et al., "Robust color-to-gray via nonlinear global mapping," ACM Transactions on Graphics (TOG) 28(5), 161 (2009).

8 C. Lu, L. Xu, and J. Jia, “Contrast preserving decolorization,” in Computational Photography (ICCP), 2012 IEEE International Conference on, 1-7, IEEE (2012).

9 C. O. Ancuti, C. Ancuti, C. Hermans, et al., "Image and video decolorization by fusion," in Asian Conference on Computer Vision, 79-92, Springer (2010). 
10 C. Ancuti and C. O. Ancuti, "Laplacian-guided image decolorization," in Image Processing (ICIP), 2016 IEEE International Conference on, 4107-4111, IEEE (2016).

11 Q. Liu, P. X. Liu, W. Xie, et al., "Gcsdecolor: gradient correlation similarity for efficient contrast preserving decolorization," IEEE Transactions on Image Processing 24(9), 28892904 (2015).

12 Y. Song and L. Gong, "O (1) contrast preserving decolorization using linear local mapping," in Wireless Communications \& Signal Processing (WCSP), 2016 8th International Conference on, 1-6, IEEE (2016).

13 S. Liu and X. Zhang, "Image decolorization combining local features and exposure features," IEEE Transactions on Multimedia (2019).

14 B. Cai, X. Xu, and X. Xing, "Perception preserving decolorization," in 2018 25th IEEE International Conference on Image Processing (ICIP), 2810-2814, IEEE (2018).

15 X. Hou, J. Duan, and G. Qiu, "Deep feature consistent deep image transformations: Downscaling, decolorization and hdr tone mapping," arXiv preprint arXiv:1707.09482 (2017).

16 Z. Lin, W. Zhang, and X. Tang, "Learning partial differential equations for computer vision," Peking Univ., Chin. Univ. of Hong Kong (2008).

$17 \mathrm{X}$. Zhang and S. Liu, "Contrast preserving image decolorization combining global features and local semantic features," The Visual Computer 34(6-8), 1099-1108 (2018).

18 Q. Liu and H. Leung, "Variable augmented neural network for decolorization and multiexposure fusion," Information Fusion 46, 114-127 (2019).

19 K. Simonyan and A. Zisserman, "Very deep convolutional networks for large-scale image recognition. 2014," arXiv preprint arXiv:1409.1556 (2018). 
$20 \mathrm{C} . \mathrm{Lu}, \mathrm{L} . \mathrm{Xu}$, and J. Jia, "Contrast preserving decolorization with perception-based quality metrics," International journal of computer vision 110(2), 222-239 (2014).

21 L.-C. Ou, M. R. Luo, A. Woodcock, et al., "A study of colour emotion and colour preference. part i: Colour emotions for single colours," Color Research \& Application 29(3), 232-240 (2004).

22 S. Niemeyer, "Ec88-423 color expression in the home," (1988).

23 C. L. Eastlake, Goethe's Theory of Colours, BoD-Books on Demand (2020).

24 V. A. Nguyen, I. P. Howard, and R. S. Allison, "Detection of the depth order of defocused images," Vision Research 45(8), 1003-1011 (2005).

25 L. F. Hodges and D. F. McAllister, "Chromostereoscopic crt-based display," in ThreeDimensional Imaging and Remote Sensing Imaging, 902, 37-45, International Society for Optics and Photonics (1988).

26 J. Hong, H. Lee, D. Park, et al., "Depth perception enhancement based on chromostereopsis in a 3d display," Journal of Information Display 13(3), 101-106 (2012).

27 M. Cadík, "Perceptual evaluation of color-to-grayscale image conversions," in Computer Graphics Forum, 27(7), 1745-1754, Wiley Online Library (2008).

28 K. Ma, T. Zhao, K. Zeng, et al., "Objective quality assessment for color-to-gray image conversion," IEEE Transactions on Image Processing 24(12), 4673-4685 (2015).

29 Z. Wang, A. C. Bovik, H. R. Sheikh, et al., "Image quality assessment: from error visibility to structural similarity," IEEE transactions on image processing 13(4), 600-612 (2004).

30 H. Z. Nafchi, A. Shahkolaei, R. Hedjam, et al., "Corrc2g: Color to gray conversion by correlation," IEEE Signal Processing Letters 24(11), 1651-1655 (2017). 
31 M. Kass and J. Solomon, "Smoothed local histogram filters," ACM Transactions on Graphics (TOG) 29(4), 100 (2010).

32 V. Chesnokov, "Image enhancement methods and apparatus therefor," (2007). US Patent $7,302,110$.

33 P. Ambalathankandy, M. Ikebe, T. Yoshida, et al., "An adaptive global and local tone mapping algorithm implemented on fpga," IEEE Transactions on Circuits and Systems for Video Technology (2019).

34 J. Xiong, H. Lu, Q. Liu, et al., "Parametric ratio-based method for efficient contrastpreserving decolorization," Multimedia Tools and Applications 77(12), 15721-15745 (2018).

35 Q. Liu, G. Shao, Y. Wang, et al., "Log-euclidean metrics for contrast preserving decolorization,” IEEE Transactions on Image Processing 26(12), 5772-5783 (2017). 


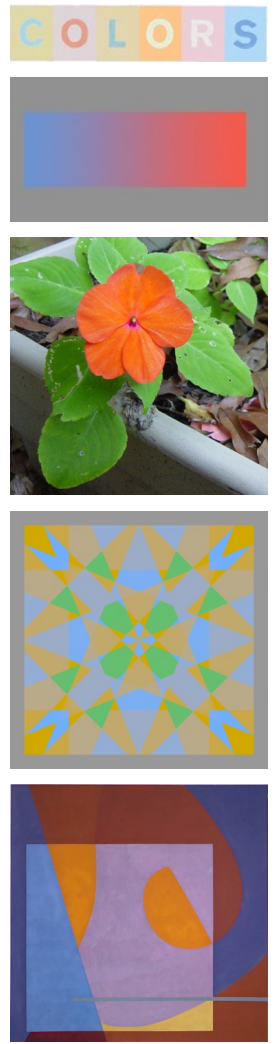

Input
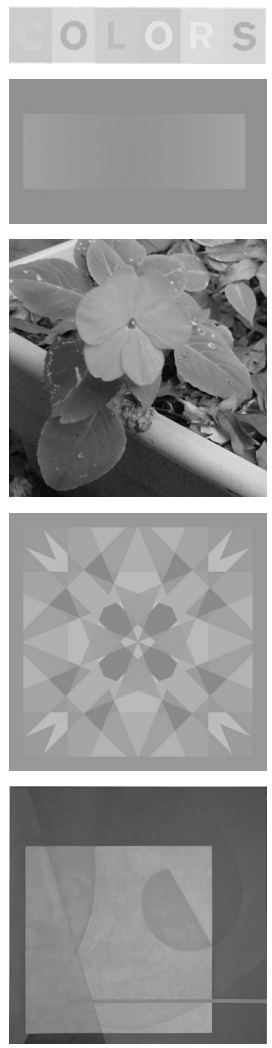

Our's
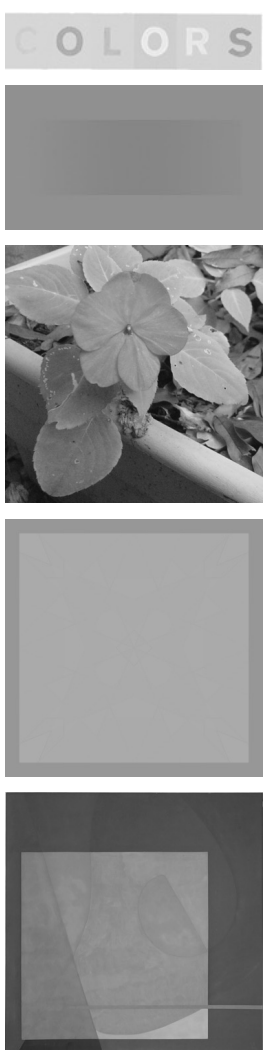

CIELAB
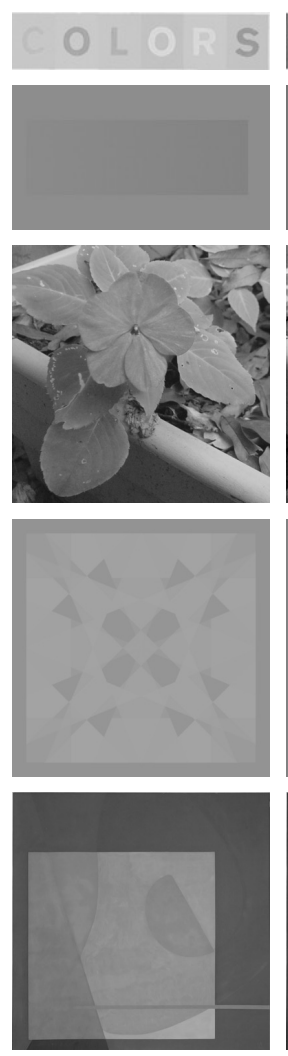

$\mathrm{YCbCr}$
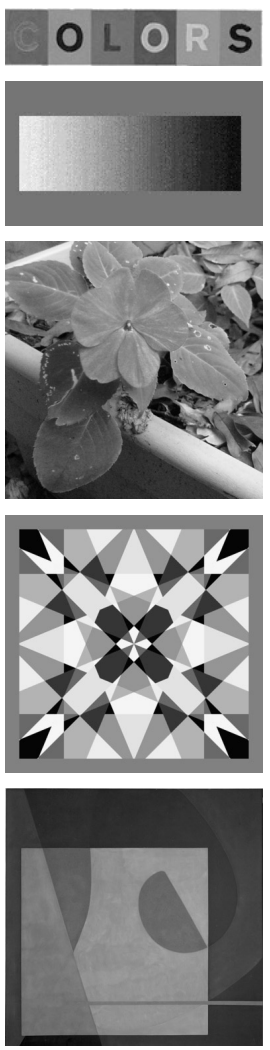

Xiong et al
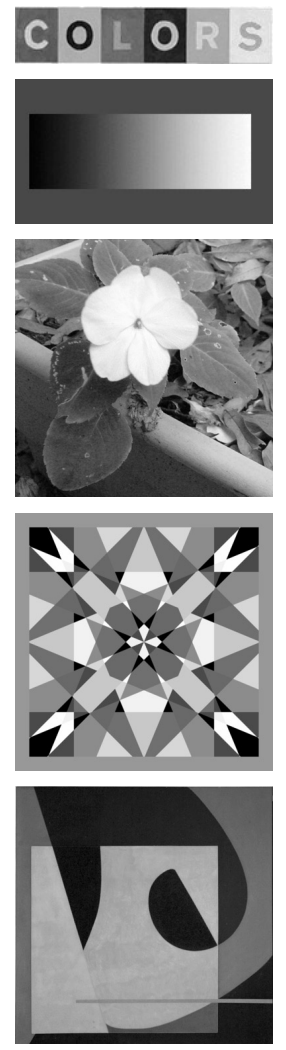

Liu et al

Fig 16 Color to gray conversion comparison using five images from Cadik's dataset. ${ }^{27}$ Decolorization methods: Ours, CIELAB, YCbCr, Xiong et al., ${ }^{34}$ and Liu et al. ${ }^{35}$ 